\title{
Challenges Faced by the Biopharmaceutical Industry in the Development and Marketing Authorization of Biosimilar Medicines in BRICS-TM Countries: An Exploratory Study
}

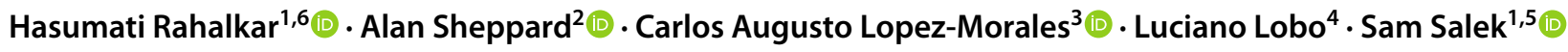

Accepted: 24 June 2021 / Published online: 22 July 2021

(c) The Author(s) 2021

\begin{abstract}
Background Biosimilars are expected to emerge as a rapidly growing segment of the biopharmaceutical industry. However, the biosimilar industry faces multiple challenges and obstacles in developing and marketing these complex products. Divergent regulatory framework in emerging countries adds to repetitive trials and increased cost of biosimilar development, delaying the approval process. Due to such roadblocks, healthcare systems and patients are yet to realize the full benefits of biosimilars.

Objectives The aim of this exploratory study was to specifically identify the challenges faced by the industry in emerging countries including Brazil, Russia, India, China, South Africa, Turkey and Mexico (BRICS-TM), pertaining to biosimilar development and the regulatory approval process. In particular, this study aims to understand the perceptions of industry on the barriers faced by them in terms of complexity, costs for biosimilar development and time-to-market for biosimilar product. Methods A semi-quantitative questionnaire was designed based on secondary research. A total of 93 industry personnel and representatives from 14 trade associations from the BRICS-TM countries with 15-year minimum experience were identified and invited to take part in the study and participate in interviews, which were recorded verbatim. Data processing and analysis was carried out; descriptive statistics were used for quantitative data and content analysis was employed to generate themes for qualitative data.

Results Of the 107 biopharmaceutical industry and trade association representatives invited to participate in the study, respondents from 33 biopharmaceutical companies agreed to take part and underwent the interviews. The industry personnel perceived biosimilar guidelines and approval processes as being protracted and in a state of evolution. The absence of an abridged approval pathway limited effectiveness of the regulatory process. The biggest hurdles in the development of biosimilar dossiers were the sourcing of the reference biological product and expectations around confirmatory clinical trials by the agencies. The non-comprehensive implementation of a stepwise approach resulting in unnecessary toxicity studies was also reported as a major challenge. The authors recommend further primary research with BRICS-TM regulatory agencies in order to propose a simplified pathway for development and approval.

Conclusions Lack of standardized biosimilar development criteria and regulatory convergence across BRICS-TM agencies has led to challenges in multi-country development programmes for these medicines, in turn impacting the ability of industry to launch newer and more affordable biosimilars.
\end{abstract}

Hasumati Rahalkar

hasumati@metinapharmconsulting.com

1 School of Life and Medical Sciences, University of Hertfordshire, Hatfield, UK

2 Ascher Resources Ltd, London, UK

3 Unidad de Desarrollo e Investigación en Bioprocesos, Mexico City, Mexico
4 Pro Genericos, The Brazilian Association of Generic Drugs, Sao Paulo, Brazil

5 Institute of Medicines Development, Cardiff, UK

6 Metina PharmConsulting Pvt. Ltd., 901-903, Goodwill Infinity, Sector 12, Kharghar, Navi Mumbai, Maharashtra 410210, India 


\section{Introduction}

Despite the high cost of therapy, the clinical efficacy and safety profile of biologic medicines has propelled huge growth of these treatments across the world [1]. Today, biologics are one of the largest and fastest growing sectors of the prescription product market with the market share of biologics growing steadily relative to small molecules. The new product pipelines of leading companies suggest that this growth dynamic will continue and be broad-based across various therapeutic areas [2].

However, the high cost of therapy with original biologics puts them out of reach for many across the world. Biosimilar medicines are usually made available at a significant discount to original biologics, and therefore have the potential to improve access and create valuable savings for patients and healthcare systems [3]. Biosimilars are products that are similar to a reference biologic product (RBP) and according to the United States Food and Drug Administration (US FDA), need to be highly similar to the approved reference product without any clinically meaningful differences in terms of safety, purity and potency [4].

When the concept of biosimilars first emerged, it generated high expectations from the access and cost savings potential that the medicines could bring to patients globally. However, due to several roadblocks, healthcare systems are yet to realize the full benefits of biosimilars [5]. Significant challenges persist from the perspective of all key stakeholders involved, that is, regulators, industry, physicians and patients.

The biosimilar industry faces multiple challenges to develop and market these complex products [6]. Compared with the well-established approval process for new chemical entities (NCEs) and small-molecule generics, the framework for approval of new biological entities (NBEs) and biosimilar products is evolving in stages across most of the developing countries. Biosimilar developers face obstacles to receiving appropriate advice, which leads to delays in product launches and late returns on investments [6]. In addition, most of the emerging market agencies have unclear regulatory processes with little global convergence. This makes global or multi-country developments expensive, lengthy and risky.

The cost to develop and gain approval for a biosimilar medicine in the US ranges between US $\$ 100$ million to US\$200 million [7]. This is significantly different from the cost of developing a small molecule generic, which typically ranges from US\$1 million to US $\$ 5$ million [7]. Biosimilar development costs are high due to greater clinical trial requirements and a need for sophisticated manufacturing facilities and cutting-edge technologies. Additionally, there is a requirement for investment in more technically skilled and competent manpower resources alongside direct promotional activities aimed at physicians and patients. Due to the inherent variability of biologics, the reproducibility of biosimilars is a big challenge and thus they are more complex to develop and manufacture [7]. The timelines for development and approval of biosimilars is also much longer than that of the small molecule generics. A United States Federal Trade Commission Report states that it takes 8-10 years to develop a biosimilar compared with 3-5 years for a small molecule generic [7].

Overall, challenges faced by the industry in complexity, costs, time-to-market and regulatory pathway for development and approval have resulted in a significant entry barrier for new players in this space. This, in turn, has led to suboptimal patient access to biosimilars [8].

The aim of this exploratory study was to specifically identify the challenges faced by the industry in Brazil, Russia, India, China, South Africa, Turkey and Mexico (BRICS$\mathrm{TM})$ pertaining to biosimilar development and the approval processes, including concerns on pricing and market access.

This study is a part of a larger research programme covering regulatory agencies, industry, physicians and patients in order to validate findings of some secondary research, published in the review article "Quality, non-clinical and clinical considerations for biosimilar monoclonal antibody development: EU, WHO, USA, Canada and BRICS-TM regulatory guidelines" [9].

\section{Methods}

A semi-quantitative questionnaire was compiled (in English), targeting various topics of concern for the biopharmaceutical industry based on information from the literature, entitled Biosimilar Development, Submission and Review (BDSR). The BDSR questionnaire consists of four parts: general information on biosimilar experience of the company; regulatory biosimilar approval process challenges and suggestions on areas of improvement; challenges pertaining to biosimilar development and suggestions on areas of improvement; and concerns faced by the industry in the area of biosimilar pricing and access (see supplementary information in the electronic supplementary material [ESM]). The industry questionnaire underwent several refinement processes, including content validation by two industry experts, in order to produce the final version.

Two target groups across the BRICS-TM countries were selected: active industry personnel with experience of over 15 years in the biosimilar space; and representatives from the pharmaceutical trade associations who have member companies with marketed biosimilar products. 
Recent estimates of companies marketing and developing biosimilar medicines range between 100 and 182 [10, 11]. Most of these companies are based in high-income, developed countries [12], with fewer active industry players in developing countries. Also, the expertise and knowledge level of most of the industry personnel in developing countries are lower. Those who declined to take part in the study offered reasons of confidentiality issues and time constraint. Industry personnel could be contacted relatively easily in India as the researcher was from the same country and had prior contacts and hence could reach the industry personnel. However, a similar network of industry personnel in the other countries was difficult to establish and therefore this could have impacted recruitment of participants from these countries. Efforts were made to improve the response rate by carrying out three follow-ups with the non-responders.

A secondary online search was performed using search terms such as biosimilar developer; biosimilar marketer; biopharmaceutical company; biosimilar approvals; trade associations; monoclonal antibodies and specific biological molecules. Sources included review articles, correspondence, meeting reports, opinions and abstracts obtained from Google scholar, websites of BRICS-TM health agencies, the University of Hertfordshire library and regulatory focus journals. The online secondary search was conducted from January 2020 to March 2020, resulting in a list of 41 biopharmaceutical companies marketing or developing biosimilars in BRICS-TM countries as well as 14 active trade associations within these countries, which included manufacturers and marketers of biosimilars.

The trade associations identified were Pharmaceutical Research Industry Association, Brazil; Pró Genéricos - Associação Brasileira das Indústrias de Medicamentos Genéricos, Brazil; Association of International Pharmaceutical Manufacturers, Russia; Association of Biotechnology Led Enterprises (ABLE), India; Indian Pharmaceutical Association (IPA); China Pharmaceutical Industry Association; Chinese Pharmaceutical Association; South Africa Association of Pharmacists in Industry; The Innovative Pharmaceutical Association South Africa (IPASA); International Generic and Biosimilar Medicines Association (IGBA) South Africa; Turkey International Trade Association; Pharmaceuticals Manufacturers Association of Turkey (IEIS); Mexican Association of Pharmaceutical Research Industries; and International Generic and Biosimilar Medicines Association (IGBA), Mexico.

A total of 93 industry personnel working within the identified companies and representatives from all 14 trade associations were invited to take part in the study. Contact details of industry personnel and trade associations were obtained through company websites, trade association websites, LinkedIn pages and through industry contacts of the authors. The study was based on an electronic questionnaire and, following completion, the participants were interviewed face-to-face (average of 45 minutes' duration) using an online platform in order to verify their responses, expand on their views and minimise bias due to misinterpretation. Confidentiality issues were cited by 14 industry personnel as a reason for non-participation and 46 did not respond at all. None of the 14 trade associations responded. In order to minimize bias arising from differences between responders and non-responders, three follow ups were carried out to try and maximize the response rate. However, this did not lead to an improved response rate (Fig. 1). Affirmative responses were received from 33 industry personnel who completed a web-based questionnaire.

Subsequently, interviews were conducted with the 33 study participants via phone call or web meetings to verify their responses, fill the gaps and provide additional comments based on their level of experience. This was carried out between March and October 2020.

\subsection{Data Processing and Analysis}

This was an exploratory study attempting to generate a hypothesis; therefore, no sample size calculation was carried out. However, the sample size may not be adequate in generalizing the results and bias could have been introduced as a result of purposive sampling. Since no statistical test was applied to the data, this removed the possibility of bias due to such tests. Data processing and analysis was carried out using Microsoft Excel and the Statistical Product and Service Solutions (SPSS) analytical software; descriptive statistics (i.e. mean, standard deviation, median, range and mode) were used for quantitative data, and content analysis was employed to generate themes and sub-themes for qualitative data.

\subsection{Ethics Approval}

The study was approved by the Health, Science, Engineering and Technology ECDA, University of Hertfordshire. Protocol number for the same is aLMS/PGR/UH/03332(1).

\section{Results}

\subsection{Demographic Characteristics of the Study Participants}

Out of the 107 personnel from the biopharmaceutical industry and representatives from trade associations contacted for the study, 33 agreed to take part. Of those that completed the study, 6 were from Brazil, 4 from Russia, 15 from India, 1 from China, 1 from South Africa, 1 from Turkey and 5 from Mexico. The respondents were senior level executives 
Collated list of 41 companies marketing/ developing biosimilar medicines in BRICS-TM countries
Collated list of 14 active biopharmaceutical trade associations in BRICS-TM, with member companies marketing biosimilar medicines

93 industry personnel and representatives from 14 trade associations were requested for interviews via emails and phone calls $(n=107)$

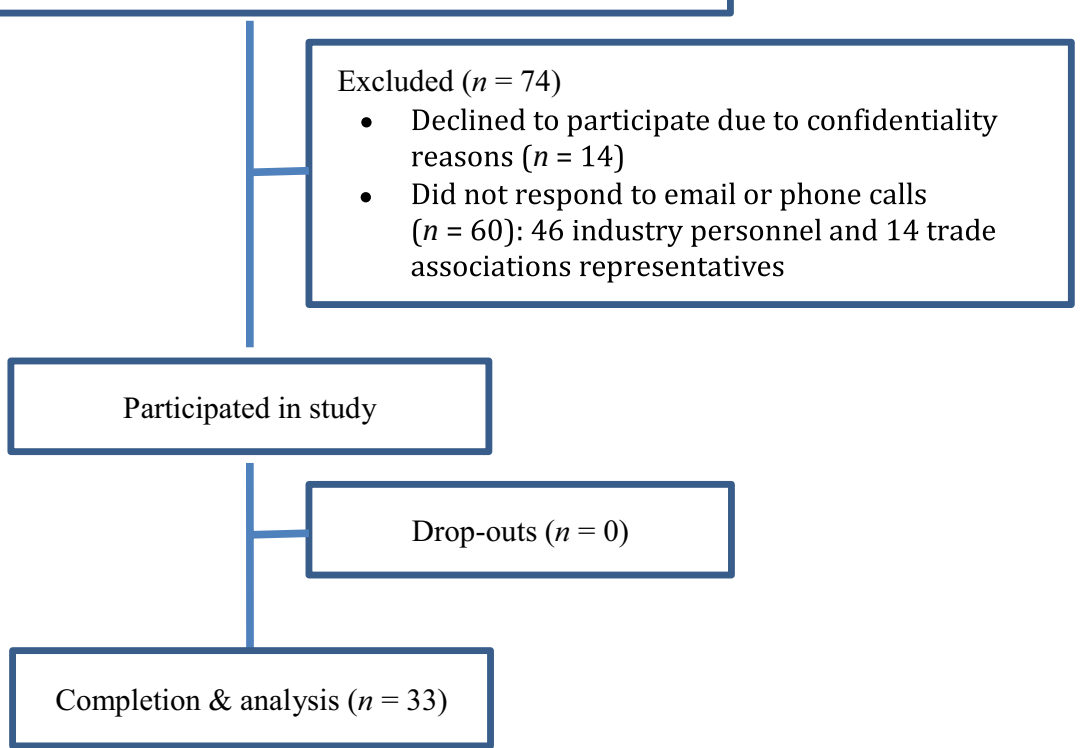

Fig. 1 CONSORT diagram. BRICS-TM Brazil, Russia, India, China, South Africa, Turkey, Mexico

with a designation of Vice President and above, representing Research and Development, Regulatory, Manufacturing and Marketing divisions of biopharmaceutical companies. The demographic characteristics of the study participants are presented in Table 1.

\subsection{Biopharmaceutical Industry of BRICS-TM}

The study participants had varied experience in the area of biosimilars. More than $58 \%$ of the participants belonged to BRICS-TM companies that have been involved in biosimilar development for more than a decade (11 years and above), while $35 \%$ of these were engaged in biosimilar development for $6-10$ years. There were only $6 \%$ of respondents whose companies had experience of $<5$ years.

Thirty $(90 \%)$ of the participating companies were marketing biosimilars. Of these, only 11 (37\%) were marketing less than three biosimilar molecules, while most have commercialized between three and ten molecules. Four (13\%) companies are marketing more than ten biosimilars in these emerging markets; $59 \%$ of these companies are developing products for commercializing in other emerging markets and $78 \%$ have an in-house biologics manufacturing facility. Therefore, the nature and characteristics of the companies that took part in this study confirmed their suitability for continuing the interview for the other parts of the questionnaire involving the challenges of biosimilar development and regulatory processes.

\subsection{Biosimilar Guidelines and Approval Process}

\subsubsection{Guidelines, Evaluation and Approval Process}

In response to a question on the guidelines and approval process for biosimilars, only $26 \%$ considered the guidelines to be well defined and transparent with an efficient review process. The guidelines were considered to be evolving with a tedious review process by $64 \%$ of respondents and the remaining $10 \%$ felt that there was a lack of clarity and transparency with guidelines subject to different interpretation, see Fig. 2. 
Table 1 Demographic characteristics of the study participants

\begin{tabular}{lllll}
\hline Function & BD & Technical & Regulatory & R\&D \\
\multicolumn{5}{l}{ operations } \\
\end{tabular}

$B D$ Business Development, $R \& D$ Research and Development, $N A$ Not Applicable

${ }^{a}$ Technical operations includes respondents who are Chief Scientific Officers or working in Manufacturing, Operations, and Quality Control departments

In India, 13 out of 15 respondents indicated an evolving and tedious regulatory process. Participants noted that the coordination between two government bodies separately reviewing non-clinical (Department of BiotechnologyDBT) and clinical data (Central Drugs Standard Control Organisation-CDSCO) has much scope for improvement. In Mexico too, three out of five respondents indicated the evolving guidelines and tedious regulatory process for biosimilars. Notably in Brazil, four out of six respondents indicated that there were well defined, transparent guidelines and an efficient review process. This could, in part, be attributed to the fact that all meetings between industry and the Agência Nacional de Vigilância Sanitária (ANVISA-the Brazil National Health Surveillance Agency) are recorded and can be retrieved and referred to. With regards to the transparency of the regulatory review process for biosimilars, $43 \%$ of BRICS-TM respondents stated that the review process was generally transparent on the main milestones but the decision-making process for each milestone was nontransparent. However, 24\% of BRICS-TM reported that the review process was non-transparent.

The participants were asked to rate (on a 5-point scale where $1=$ low concern and $5=$ significant challenge) the key challenges in the review and evaluation of biosimilar dossiers by the respective country's regulatory agency. It is notable that all the identified challenges were rated 3 (moderate challenge) and above, indicating that these were all significant issues across the countries (Fig. 3). 'Process inefficiency' emerged as the single highest concern with a median rating of 4 and a mode value of 5. 'Inadequate communication channel between the industry and the agency' and 'Lack of consultation with applicant company' emerged as the second biggest concerns with a median rating of 3.5 and a mode value of 4 for both parameters.

Some of these challenges could be mitigated by the timely provision of appropriate scientific advice from the agency to the company. However, in response to a question on this matter, it appeared that about $60 \%$ of respondents across BRICS-TM either did not receive advice or received advice that was inadequate. This concern is more pressing in Russia where there is no possibility of interaction with the Ministry of Health $(\mathrm{MoH})$ on this subject. Notably, all respondents from Brazil confirmed provision of adequacy of scientific advice from ANVISA.

\subsubsection{Efficiency and Effectiveness of Approval Process}

The approval timelines of biosimilar applications did not emerge as a significant challenge with the BRICS-TM agencies. Most regulatory agencies have an average timeline of 24 months, with CDSCO India and MoH Russia having a shorter timeline of 6-12 months. In Mexico, the average approval timelines by Comisión Federal para la Protección contra Riesgos Sanitarios (COFEPRIS) was reported as $9-12$ months by the respondents. In India specifically, the most frequent timeline was reported as being 6 months. The expected optimal approval timelines by companies were reported to be in the range of 9-12 months across the BRICS-TM countries, with 9 months being the most frequently reported. The largest gap between the agency practice and the industry expectation occurred in Brazil (12 


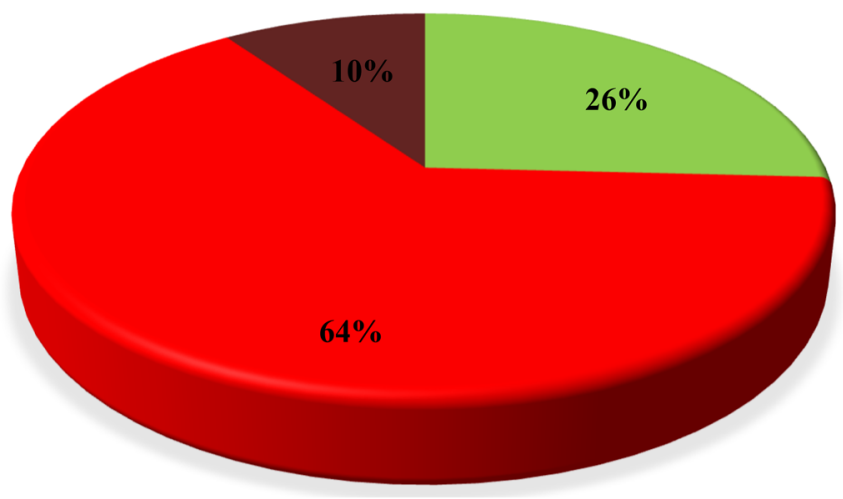

Well-defined, transparent guidelines and efficient review process

Evolving guidelines with tedious review process

Lack of clarity and transparency, with guidelines subject to different interpretations

*all percentages have been rounded off to nearest whole number

Fig. 2 Industry feedback on guidelines and approval process

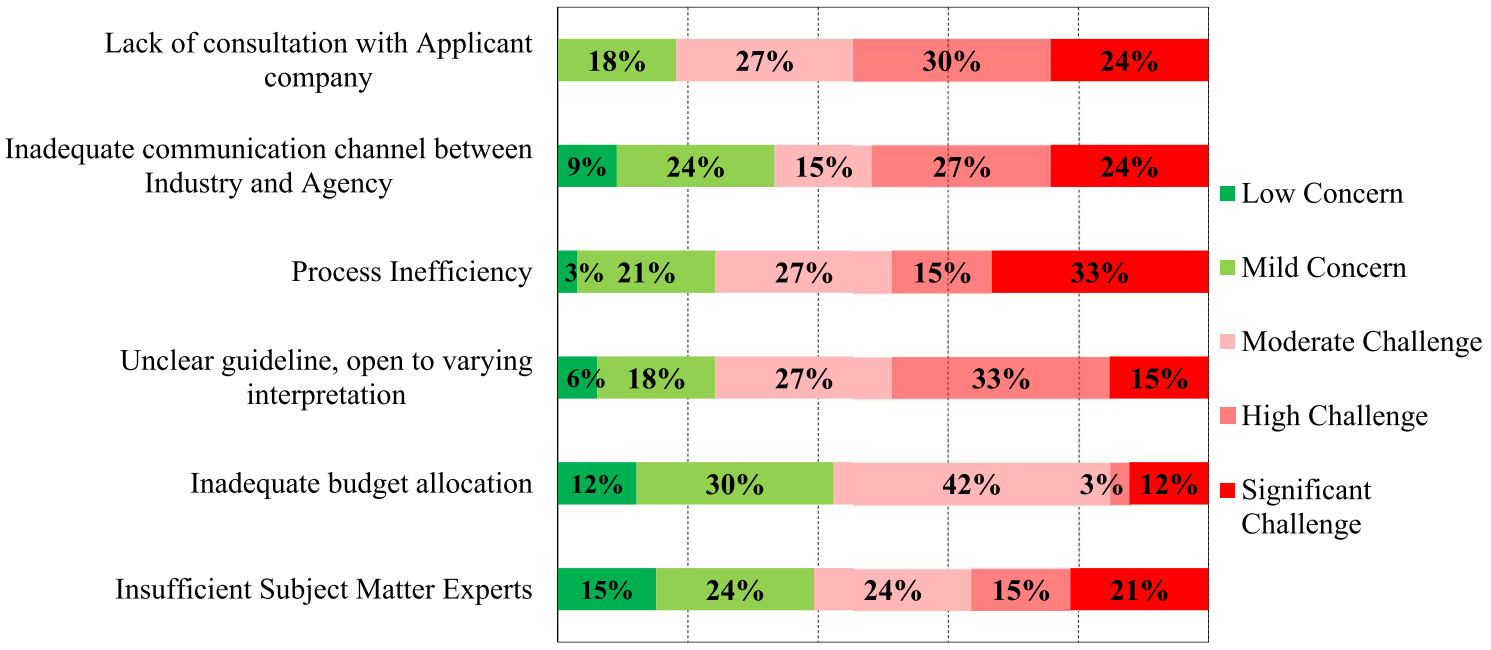

*all percentages have been rounded off to nearest whole number

Fig. 3 Challenges in review and evaluation of biosimilar dossier

months). The study participants representing the BRICSTM countries highlighted an absence of an abridged review pathway, although in Mexico, $80 \%$ of respondents indicated an abridged review pathway being followed by their agency. In response to a question on fast-track approvals of biosimilars, $55 \%$ of respondents reported that such procedures exist. However very few have had success in availing of such approvals, except in the case of orphan drugs and recently in the case of medicines for the treatment of COVID-19 (e.g., itolizumab was approved by CDSCO India for restricted emergency use in Acute Respiratory Distress Syndrome [ARDS] caused by COVID-19, in July 2020. This monoclonal antibody was approved in India in 2013, and the additional indication was approved via a fast-track procedure).

Most countries require pricing approval for biosimilars before commercialization. This process is not unduly long with timelines ranging from 2 to 6 months.

\subsection{Development Parameters}

\subsubsection{Reference Biologic Product (RBP) Selection}

The BRICS-TM agencies provide clarity in terms of the RBP to be used for each biosimilar development and the list of countries from which sourcing of a foreign acceptable comparator (FAC) is admissible. 
While the industry may have clarity around the reference product and the country from which it is expected to be sourced, sourcing of the RBP remains a key challenge.

3.4.1.1 Multiple Lots of RBP In order to meet the requirement for statistical justification of analytical similarity of the biosimilar with the original biologic, multiple lots of the reference product need to be sourced, as reported by 29 (88\%) of the participants. The exact number of batches are often not specified and may vary from case to case, and it is open to industry interpretation. Individual responses from within countries vary widely but the frequency analysis revealed a mode of 3-10 batches of different ages except South Africa, where it was indicated there was no expectation for multiple batches. The sourcing of multiple batches of the RBP is a significant challenge for industry, as indicated by $70 \%$ of the companies. Content analysis of the participants' comments showed that concurrent availability of multiple lots of the RBP in the market at any given point of time is a key hurdle.

3.4.1.2 Quantity of RBP Even if available, the quantity of product required per batch for various tests and studies (characterizations, comparability studies, clinical studies) can be difficult to source. Large quantities of RBP required to perform comparative clinical safety and efficacy studies increases the overall cost of development.

3.4.1.3 RBP from a Single Drug Substance The innovator usually manufactures the drug substance on a large scale. Therefore, multiple product batches can come from a single drug substance, which complicates the efforts of biosimilar developers to get drug product batches from different drug substances. Sourcing multiple batches of the same biological substance leads to limited variation in analytical results, which in turn complicates the justification for product specifications with the regulator. In order to remedy such a situation, the biosimilar manufacturer often has to set up stringent analytical specifications, which subsequently results in manufacturing difficulties, such as non-compliance to stringent standards.

3.4.1.4 Change in Manufacturing Process of RBP If the innovator decides to change the manufacturing process and obtains approval for the same, a fresh development process with new batches needs to be initiated by the biosimilar manufacturer.

3.4.1.5 RBP Non-Availability in Open Market In Russia, a specific issue is that all biologics are procured directly by the government from the distributors, resulting in no availability of the product in the open market for procurement. Consequently, all these factors result in sizable time and cost escalation for the companies developing biosimilars.

\subsubsection{Criteria for Biosimilarity}

Criteria for biosimilarity encompasses comparative physico-chemical and biological characterization, in vitro nonclinical studies, in vivo safety data and confirmatory clinical safety and efficacy studies.

In response to a question on challenges to prove biosimilarity, the study participants from BRICS-TM rated 'confirmatory clinical safety and efficacy study' as their highest concern with a mean rating of 2.4 on a scale of 1 to 3 . The most frequent rating value was 3 with 22 of 33 respondents rating this as a 'significant challenge'. The mean rating of other parameters included 'comparative physico-chemical and biological characterization (quality)'; and 'in vitro or in vivo non-clinical study', which were rated as 2 (i.e., moderate challenge), but not as high as 'confirmatory clinical safety and efficacy study' (Fig. 4).

In terms of efficacy and safety studies, the study participants reported that the cost of trials, large sample size for trials, lead time for patient recruitment and significant drop out rates were some of the major operational issues in conducting clinical studies for biosimilars. Companies also face lack of expertise to develop in-house bioassay methods for biosimilars.

\subsubsection{Naming of Biosimilars}

The BRICS-TM regulatory agencies mandate the same international non-proprietary name (INN) for biosimilars as the RBP, as reported by 30 (90\%) of the companies participating in the study. This is different from the approach followed by the US FDA, which expects the nomenclature to be in accordance with 'Guiding Principles for Coining United States Adopted Names for Drugs' [13] for each biologic and biosimilar [14] (Table 2).

\subsubsection{Non-Clinical Studies}

As reported earlier in this article, non-clinical studies for biosimilars were not rated as a significant challenge by the study participants from the BRICS-TM countries. Further details from the responses indicated that though non-clinical studies data are mandatory as part of the application, no regulatory agency mandates that studies be performed locally. Content analysis of the free-text comments proposed that the regulatory agencies should move towards a step-wise approach to development and mandate non-clinical data only if absolutely required. 


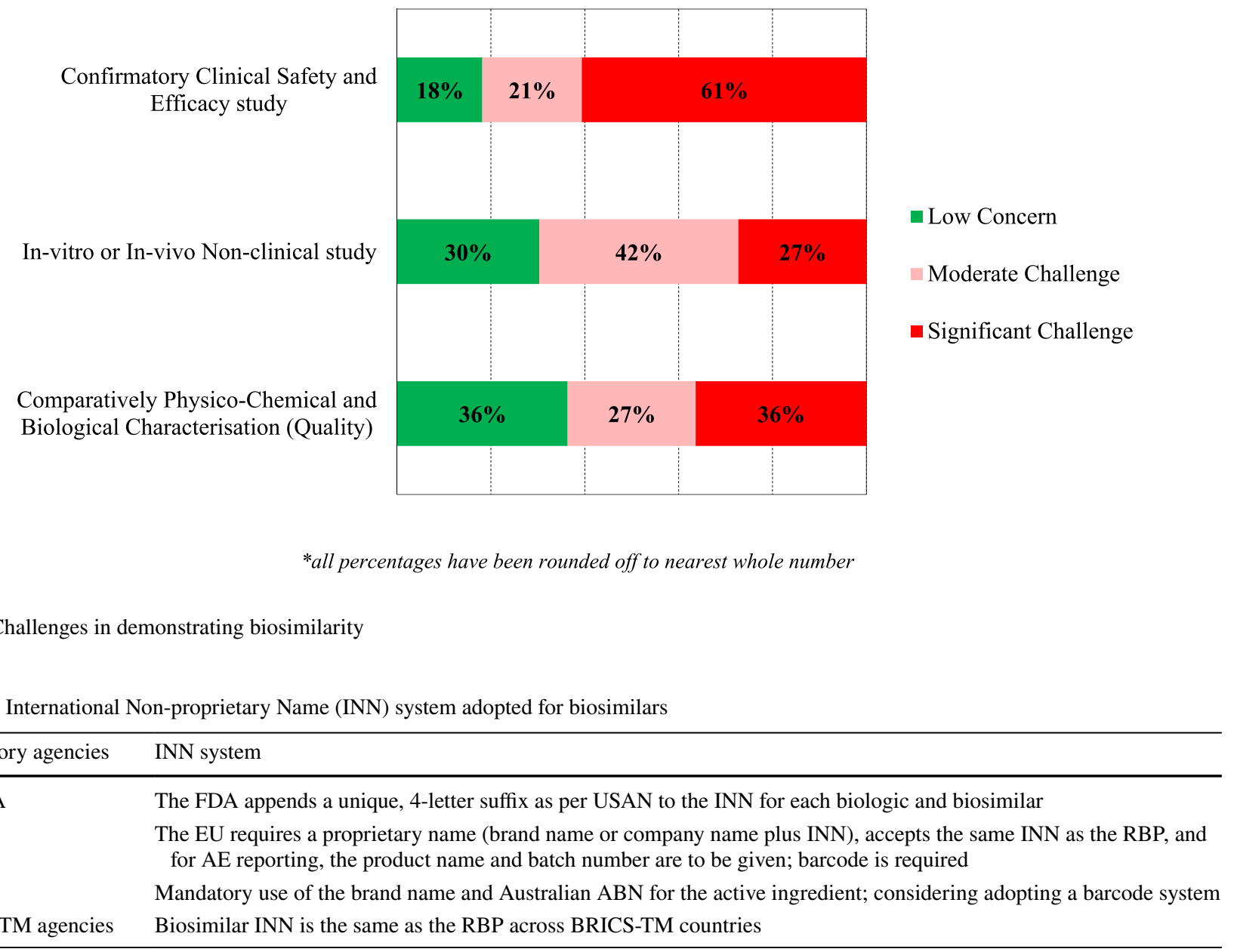

Fig. 4 Challenges in demonstrating biosimilarity

Table 2 International Non-proprietary Name (INN) system adopted for biosimilars

\begin{tabular}{ll}
\hline Regulatory agencies & INN system \\
\hline US FDA & The FDA appends a unique, 4-letter suffix as per USAN to the INN for each biologic and biosimilar \\
EMA & $\begin{array}{l}\text { The EU requires a proprietary name (brand name or company name plus INN), accepts the same INN as the RBP, and } \\
\text { for AE reporting, the product name and batch number are to be given; barcode is required } \\
\text { Mandatory use of the brand name and Australian ABN for the active ingredient; considering adopting a barcode system } \\
\text { TGA }\end{array}$ \\
$\begin{array}{l}\text { BRICS-TM agencies } \\
\text { Biosimilar INN is the same as the RBP across BRICS-TM countries }\end{array}$
\end{tabular}

$A B N$ Approved Biological Name, AE Adverse Events, BRICS-TM Brazil, Russia, India, China, South Africa, Turkey, Mexico, EMA European Medicines Agency, EU European Union, RBP Reference Biologic Product, TGA Therapeutic Goods Administration, USAN United States Adopted Names, US FDA United States Food and Drug Administration

\subsubsection{Clinical Studies}

As part of the biosimilar application, most agencies expect data from a phase I study (comparative clinical pharmacokinetic [PK], pharmacodynamic [PD] or combined PK/PD), phase III study (comparative clinical safety and efficacy) and phase IV post-marketing surveillance study (including follow-up study for immunogenicity). A comparative confirmatory clinical study (phase III) is one of the most important requirements to be fulfilled as part of the marketing authorization of the application. In Sect. 3.3.2 of the Results describing criteria for demonstrating biosimilarity, it was reported that the companies taking part in this study rated 'confirmatory clinical safety and efficacy study' as the highest concern for them in the development of a biosimilar product.

3.4.5.1 Mandatory Local Studies This study needs to be performed locally in India, Mexico, Russia and China and should be comparative in nature; the reference drug needs to be used throughout the study to prove comparative efficacy of the respective biosimilar. Mostly biosimilar applicant companies combine the confirmatory clinical and immunogenicity studies.

3.4.5.2 Lack of Clarity on Study Design There exists lack of clarity on certain aspects of these studies, such as study types, population, end points, design, paediatric population, safety pharmacology expectations and follow-up period.

3.4.5.3 Other Factors The industry also faces challenges in the following areas:

1. Approval of clinical trial protocol by the regulatory agency.

2. Ethics approval.

3. Patient recruitment.

4. Availability of a clinical research organization (CRO).

5. Lack of specific and binding scientific advice on clinical studies. 
These factors were rated by the company participants (Fig. 5) across the BRICS-TM, which showed 'Lack of specific and binding scientific advice on clinical study design' to be the single biggest challenge with a mean value of 3.5 and a mode value of 4 (high challenge). 'Approval of protocol by the agency' and 'Patient recruitment' were rated as the next biggest obstacles. This outcome further validates earlier reported data on inadequacy of scientific advice.

3.4.5.4 Sample Size for Clinical Studies The BRICS-TM [15-21] agencies require confirmatory clinical studies to be performed in two arms with a patient ratio of $1: 1$ for the test and reference product. The response from countries was markedly variable; however, it was reported that the minimum expectation from the regulatory agencies was 100-200 patients per arm, or based on statistical powering of the trial, whichever was highest.

3.4.5.5 Multi-Country Development Considering the time and cost involved in developing and marketing biosimilars, the companies active in this space are those that overcome steep entry barriers and are therefore typically large multinational players. Despite this, industry players find it difficult to develop a global regulatory strategy for biosimilars covering the emerging markets, due to several hurdles. While $69 \%$ of companies indicated that they are pursuing multicountry biosimilar developments including for BRICS-TM countries, they face obstacles on several fronts (Fig. 6).

In response to a question on these challenges, the participants rated 'Lack of harmonized guideline for biosimilar development across BRICS-TM' and 'Absence of common clinical trial design and approval process across BRICS-TM' as the highest concerns, with a mean of 3.5 on a scale of 1-4 and a mode value of 4 for both. Other issues such as 'Acceptance of reference biological product across BRICS-TM' and 'Acceptance of foreign patients' data' were also rated as a 'significant challenge', with 4 being the most prevalent rating, signifying all four criteria as critical barriers.

\subsection{Pricing and Market Access Concern}

A Quintiles and IMS Health (IQVIA) article on biosimilars and biobetters published in September 2020 [22] reported that the rest of the world (RoW) countries accounted for US $\$ 0.1$ billion sales for follow-on biologics (FOBs) including biocomparables versus US $\$ 15$ billion global sales, as of second quarter 2020. It was concluded that there is an overall low coverage of biologics, and FOBs are an emerging sector where sales remain low. Further, on their own, RoW countries are unable to justify the cost of development for biosimilar projects [22].

This study delved into the reasons for limited access for patients and entry barriers for the industry. 'Innovator patent term and strategy' and 'Higher cost of therapy of biosimilars as compared to small molecule medicines' were rated as the highest barriers to access with a mean rating of 3.6 and 3.4, respectively. The next biggest obstacles were rated to be 'Challenges pertaining to regulatory framework for development and approval of biosimilars' and 'Less numbers of active industry players in biosimilar segment'. Apart from the concerns around patent terms of original biologics,

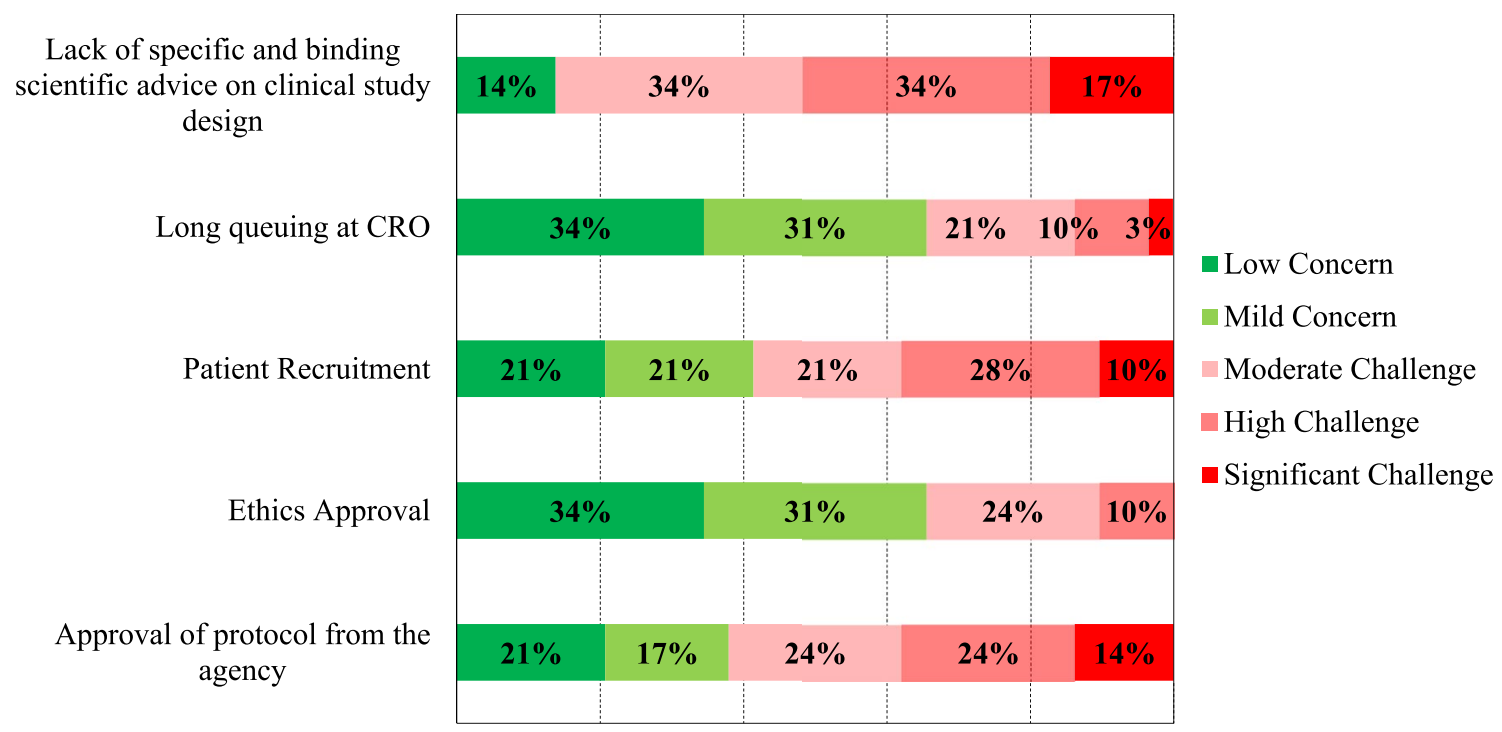

*all percentages have been rounded off to nearest whole number

Fig. 5 Challenges in clinical study. CRO Clinical Research Organization 


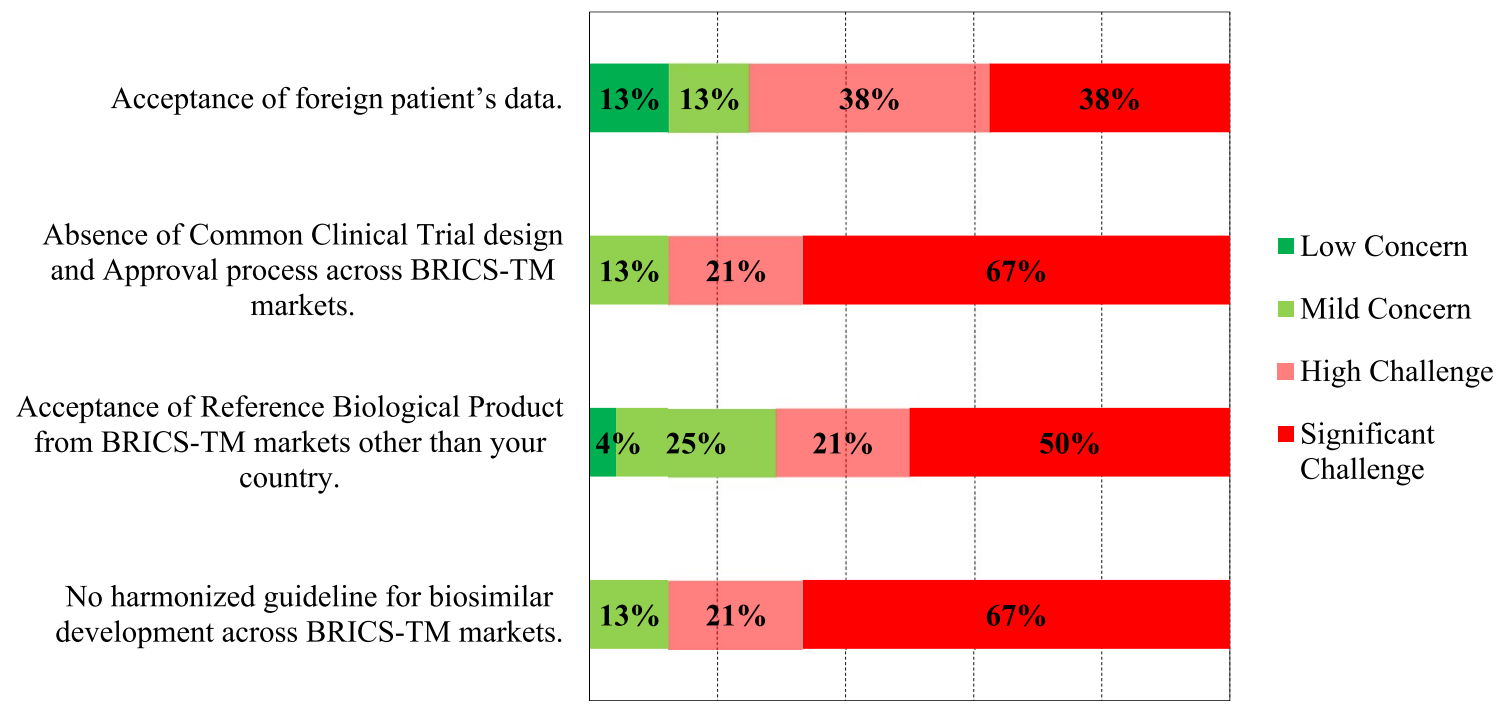

*all percentages have been rounded off to nearest whole number

Fig. 6 Development challenges in BRICS-TM market. BRICS-TM Brazil, Russia, India, China, South Africa, Turkey, Mexico

the other three challenges can be substantially mitigated by facilitating ease of development and approval of global biosimilars and enabling more competition in this space. Related to this, companies were asked about specific issues that acted as entry barriers into this space. 'Late and unsure return on investment considering high cost involved' and 'Prohibitive cost of clinical trials for biosimilars' were rated as the highest challenges with a median rating of 4.3 and 4.1 , respectively. 'Lack of in-house expertise and infrastructure in biosimilars' and 'Pressure on pricing from health authorities/insurers/procurement authority' were rated as the second-highest barriers with a median rating of 3.5 and 3.4, respectively (Fig. 7).

Pressure on pricing from Health Authorities/Insurers /Procurement Authority

Highly competitive and fluctuating prices for biosimilar

Late and unsure return on investment considering high cost involved

Prohibitive cost of Clinical Trials for biosimilar, which will need to be replicated across each BRICS-TM market

In-house expertise and infrastructure for biosimilar

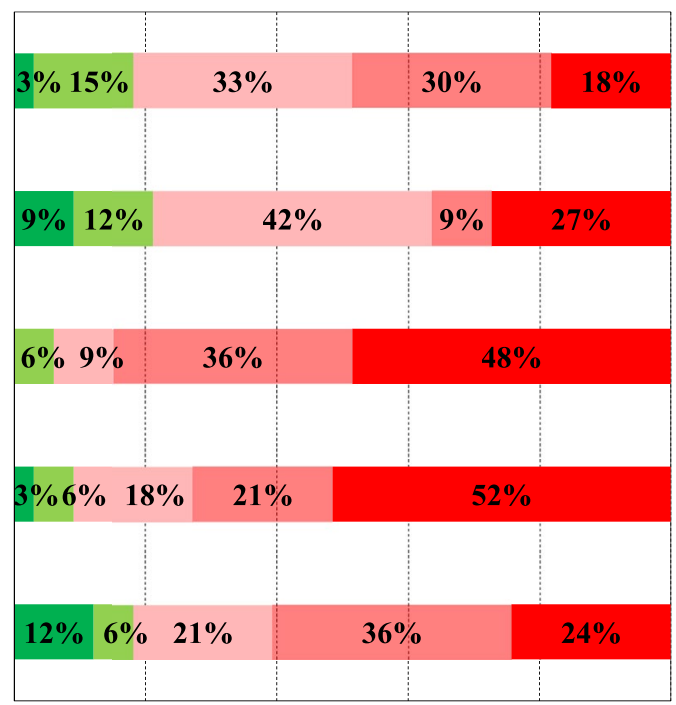

- Low Concern

- Mild Concern

Moderate

Challenge

High Challenge

Significant

Challenge

*all percentages have been rounded off to nearest whole number

Fig. 7 Entry barriers for industry players to be active in biosimilar space. BRICS-TM Brazil, Russia, India, China, South Africa, Turkey, Mexico 


\section{Discussion}

The biopharmaceutical industry faces several challenges in the development and registration of biosimilars in BRICSTM countries. The result of this exploratory research has highlighted key issues, which are summarized in Table 3. Clearly, the results indicate that across the BRICS-TM countries, the different functions were mostly aligned in their perception of the specific challenges faced by them (Table 4).

Biosimilar regulations vary widely across the regulatory agencies in emerging economies, with a patchwork of applicable rules [23]. In this research, only $26 \%$ of respondents found the available guidelines transparent and the majority felt that communication between industry and the regulatory agency is inadequate and unreliable. Process inefficiencies can often be attributed to the lack of expert resources within the biologics departments of the regulatory agencies [24]. However, building capacity and expertise in a national regulatory authority is a long-term process and quick resolutions lie in relying on information from other regulatory authorities or joint or abridged review models [25]. Also, transparency in the regulatory evaluation process would greatly contribute to establishing confidence within the industry and the recording and retrieval of meetings as followed by ANVISA is an example of 'good review practice' that can be emulated.

The RBP sourcing comes across as a major hurdle for most companies [25]. A greatly simplified basis for selecting a reference comparator, that does not require conducting new bridging studies, has been proposed and justified based on the relevant scientific data in an opinion paper [26]. In an article on 'The importance of Global Regulatory Harmonization for Biosimilar Medicines' by the IGBA [27], use of a global comparator product and waiving of bridging studies is highlighted as a key proposal to enable multi-country development. A World Health Organisation (WHO) survey result of 20 countries also reiterated these challenges [28]. A common approach to the RBP definition, harmonized expectations for the number of batches to be used for analytical similarity, acceptance to source RBP across BRICS-TM countries and establishment of an independent agency to supply RBP with varied drug substance lots in the absence of the product in the open market will enable multi-country development.

A key improvement area for regulatory agencies in the emerging economies could be adoption of a 'step-wise' approach for biosimilar development [24]. This will reduce the unnecessary non-clinical studies in cases where there is proven similarity in physicochemical characterization between the test and reference product.
Table 3 Summary of critical challenges identified, based on primary research

\begin{tabular}{|c|c|}
\hline Aspects & Critical challenges identified \\
\hline Guidelines, evaluation and approval process & $\begin{array}{l}\text { Evolving guidelines with tedious review process } \\
\text { Process inefficiency } \\
\text { Inadequate communication channel with agency }\end{array}$ \\
\hline Process effectiveness & Absence of abridged review pathway \\
\hline Development parameters & $\begin{array}{l}\text { Reference biologic product } \\
\text { - Sourcing of multiple lots within stipulated } \\
\text { timeframe } \\
\text { - Sourcing acceptable from limited countries } \\
\text { - Large quantity required for characterization } \\
\text { and clinical study } \\
\text { - Availability of RBP from different drug- } \\
\text { substance lots } \\
\text { - Cost of RBP } \\
\text { Non-clinical studies } \\
\text { - Mandatory in vitro/in vivo studies, often not } \\
\text { justified } \\
\text { Confirmatory clinical safety and efficacy studies } \\
\text { - Lack of specific and binding scientific advice } \\
\text { - Lack of harmonized guideline for biosimilar } \\
\text { development across BRICS-TM } \\
\text { - Absence of common clinical trial design and } \\
\text { approval process across BRICS-TM }\end{array}$ \\
\hline Market access and pricing & $\begin{array}{l}\text { Late and unsure return on investment consider- } \\
\text { ing high cost involved } \\
\text { Prohibitive cost of clinical trials for biosimilars } \\
\text { Pressure on pricing from health authorities/ } \\
\text { insurers/ procurement authority }\end{array}$ \\
\hline
\end{tabular}

BRICS-TM Brazil, Russia, India, China, South Africa, Turkey, Mexico, RBP Reference Biological Product 
Table 4 Breakdown of challenges in biosimilar development and approval process in BRICS-TM according to functions of the study participants

Identified challenges

Country

Prevalence of responses $(\%)$

Regulatory affairs (RA)

Evolving guidelines with tedious review process

Confirmatory clinical safety and efficacy studies

Reliance and absence of abridged review pathway

Research \& development (R\&D)

Mandatory non-clinical studies (in vitro and in vivo studies)

Lack of specific and binding scientific advice

Lack of harmonized guideline for biosimilar development across BRICS-TM

\begin{tabular}{|c|c|c|}
\hline Brazil & 33.3 & Low concern \\
\hline Russia & 50 & High concern \\
\hline India & 60 & High concern \\
\hline China & NA & NA \\
\hline South Africa & 100 & High concern \\
\hline Turkey & 0 & No concern \\
\hline Mexico & 100 & High concern \\
\hline Brazil & 66.67 & High concern \\
\hline Russia & 100 & High concern \\
\hline India & 80 & High concern \\
\hline China & NA & NA \\
\hline South Africa & 100 & High concern \\
\hline Turkey & 100 & High concern \\
\hline Mexico & 0 & No concern \\
\hline Brazil & 33.33 & Low concern \\
\hline Russia & 50 & High concern \\
\hline India & 100 & High concern \\
\hline China & NA & NA \\
\hline South Africa & 0 & No concern \\
\hline Turkey & 0 & No concern \\
\hline Mexico & 0 & No concern \\
\hline Brazil & NA & NA \\
\hline Russia & NA & NA \\
\hline India & 100 & High concern \\
\hline China & NA & NA \\
\hline South Africa & NA & NA \\
\hline Turkey & NA & NA \\
\hline Mexico & 100 & High concern \\
\hline Brazil & NA & NA \\
\hline Russia & NA & NA \\
\hline India & 100 & High concern \\
\hline China & NA & NA \\
\hline South Africa & NA & NA \\
\hline Turkey & NA & NA \\
\hline Mexico & 100 & High concern \\
\hline Brazil & NA & NA \\
\hline Russia & NA & NA \\
\hline India & 100 & High concern \\
\hline China & NA & NA \\
\hline South Africa & NA & NA \\
\hline Turkey & NA & NA \\
\hline Mexico & 100 & High concern \\
\hline
\end{tabular}

Further in the development cycle, companies have singled out the confirmatory clinical safety and efficacy study as a major concern. A McKinsey report on biosimilars has reiterated that discussion around lowering development costs via innovation is essential to ensure a sustainable future [29]. Therapeutic equivalence trials account for at least $75 \%$ of 
Table 4 (continued)

Identified challenges

Country

Prevalence of

Level of con-

responses $(\%)$

cordance of the

responses

Business development (BD)

Late and unsure return on investment considering high cost involved

Sourcing of multiple RBP lots

Pressure on pricing from Health Authorities/Insurers/Procurement Authority

Technical operations

In-house expertise and infrastructure

Submission and commercialization of three batches of validation

\begin{tabular}{|c|c|c|}
\hline Brazil & 100 & High concern \\
\hline Russia & NA & NA \\
\hline India & 100 & High concern \\
\hline China & 100 & High concern \\
\hline South Africa & NA & NA \\
\hline Turkey & NA & NA \\
\hline Mexico & NA & NA \\
\hline Brazil & 100 & High concern \\
\hline Russia & NA & NA \\
\hline India & 80 & High concern \\
\hline China & 0 & No concern \\
\hline South Africa & NA & NA \\
\hline Turkey & NA & NA \\
\hline Mexico & NA & NA \\
\hline Brazil & 100 & High concern \\
\hline Russia & NA & NA \\
\hline India & 60 & High concern \\
\hline China & 100 & High concern \\
\hline South Africa & NA & NA \\
\hline Turkey & NA & NA \\
\hline Mexico & NA & NA \\
\hline Brazil & NA & NA \\
\hline Russia & 50 & High concern \\
\hline India & 100 & High concern \\
\hline China & NA & NA \\
\hline South Africa & NA & NA \\
\hline Turkey & NA & NA \\
\hline Mexico & NA & NA \\
\hline Brazil & NA & NA \\
\hline Russia & 50 & High concern \\
\hline India & 75 & High concern \\
\hline China & NA & NA \\
\hline South Africa & NA & NA \\
\hline Turkey & NA & NA \\
\hline Mexico & 50 & High concern \\
\hline
\end{tabular}

If $\%$ outcome of respondents is $\geq 50=$ high concern, $<50=$ low concern, $0=$ no concern

BRICS-TM Brazil, Russia, India, China, South Africa, Turkey, Mexico, NA Not Applicable, RBP Reference Biological Product

total development costs [29]. Secondary research on biosimilar approvals has revealed that usually no submission gets rejected following a full review due to a finding of clinical inequivalence between the biosimilar and its RBP if the two products have been found to be highly similar in analytical and PK studies [30]. Hence, powered efficacy studies of these biosimilar candidates are of questionable value [30]. Moreover, repetition of these studies across countries and non-acceptance of foreign patients' data leads to escalation of cost and timelines, jeopardizing the return on investment for the developer. A policy paper from IGBA [31] has also reiterated the higher relevance of advanced analytical science to prove comparability in place of confirmatory clinical data. In addition, WHO is also well positioned on this subject in its 'Guidelines on evaluation of similar biotherapeutic products (SBPs)' [32], based on which other regional 
guidelines for biosimilars have been modelled into. The WHO [32] clearly notes that the demonstration of comparability of an SBP to its RBP in terms of quality (comparability exercise) is a prerequisite for the reduction of the non-clinical and clinical data set required for licensure. The guidance further elaborates its stand on abbreviated clinical development programmes for biosimilar products, citing the advancement in the development of analytical methodology for characterizing complex biotherapeutic products including monoclonal antibodies.

These barriers in the biosimilar space in emerging markets have led to relatively low patient access to biosimilar medicines when compared to developed markets [6]. Patients in these markets stand to gain the greatest increase in access as a result of biosimilar competition. There has been a marked push for high-quality biosimilars [6] but a real change will only be seen once the regulatory agencies take concrete steps towards improving efficiency and transparency of the processes, standardization of RBP requirements, establishing abridged review pathways, following a step-wise approach and accepting advanced analytical comparability data in lieu of confirmatory clinical studies.

Regulators are conservative by inclination and incentivization, and are not minded to lead progressive change, even though it may lead to improvements for patients. It is, therefore, up to industry, which invented the products and their associated scientific underpinnings in the first place, to educate regulators on efficient review pathways. It is evident from this paper that many of the regulators essentially consider biosimilars to require only a slight variation of the traditional innovator's drug review pathway, which is to deny completely the science and logic of biosimilarity and the benefits that it can confer. Requirements for approval of biosimilars based upon a good scientific understanding will find no place for studies in animals, no place for local clinical studies, few reasons to 'bridge' a local version of the reference to a version approved in another jurisdiction and no reason to require clinical equivalence studies routinely, but it is for industry to bring these understandings to patients, payers, regulators and physicians alike.

\subsection{Hypothesis to be Tested in Future Studies}

- Removal of mandatory compliance with confirmatory clinical safety and efficacy studies would improve patients' access to biosimilars.

- Adoption of verification and abridged regulatory review models would reduce the approval timelines.

- Establishment of harmonized biosimilar regulatory guidelines for the BRICS-TM countries will enhance development and approval timelines.

- Flexibilities in sourcing of RBP will result in development of a common biosimilar development programme.

\section{Conclusions}

This study delves into the understanding and perception of the respondents regarding the efficiency and effectiveness of current regulatory processes for biosimilar products and gathers suggestions on potential improvements in this area. The study was based on the experience and expertise of those involved in the research and development of biosimilars and provides a unique insight into the successes and challenges faced by the biosimilar industry at large. The findings suggested that the BRICS-TM industry faces significant challenges related to cost of development, efficiency and transparency of processes, standardization of RBP requirements, availability of clear scientific advice and acceptance of advanced analytical comparability data in lieu of confirmatory clinical studies.

Despite availability of the Similar Biotherapeutic Products (SBP) guidelines issued by WHO [32-34], findings of this study indicate that the guidelines have been only partially implemented by the National Regulatory Agencies (NRAs) of BRICS-TM countries, leaving their review practices open to interpretation. Hence, continued efforts towards a globally consistent approach to biosimilar development and approval processes considering the regional differences in regulations appears to be essential. This can be ensured by following the concepts of a 'step-wise approach' and 'head-to-head' biosimilarity, which are the core of the regulatory guidelines of mature agencies such as the European Medicines Agency (EMA), the US FDA and WHO [35]. Such global adoption of regulatory guidelines modelled over existing templates could further expedite approval and facilitate patients' access to these medicines. To achieve this, and to gain the trust and acceptance of biosimilars globally, education plays a crucial role [35]. Moreover, the role of the biopharmaceutical industry is very significant in balancing the need to account for regulatory variations against the costs of the studies required to seek biosimilar approvals in all geographic regions [36]. An in-depth knowledge of each region, early strategic planning, and effective communication with regulatory agencies would be advantageous in achieving this [37]. Further, the understanding by new inexperienced manufacturers developing biosimilar products requires great care and attention regarding the development and production of these biological products and adhering to Good Manufacturing Practice (GMP) is essential. Also, the role of NRAs in overseeing these developments through GMP inspections is critical [38]. Thus, there is an immediate need to create a culture of quality within the organization to meet the challenges posed by complex biosimilar molecules [39]. This would enable manufacturers to provide consistent production and quality control, thereby preventing 
drift from the required specifications over time and would greatly influence the acceptance of biosimilars and their integration into daily practice [40].

The findings of this study have led to a number of recommendations that it is hoped will be considered by the BRICS-TM regulatory agencies:

1. The regulatory agencies in the BRICS-TM countries should consider timely provision of appropriate tailor-made and binding scientific advice to companies engaged in biosimilar development. This should also be extended to clinical studies, if applicable.

2. The regulatory agencies should consider adopting shared evaluation, reliance and abridged regulatory review models for biosimilars.

3. The regulatory agencies should consider moving towards a step-wise approach to development and mandate non-clinical data only in cases where it is fundamentally required.

4. The regulatory agencies should consider accepting advanced analytical comparability data in lieu of confirmatory clinical studies.

5. Regulatory agencies should consider accepting RBP sourcing from BRICS-TM countries other than their own, with a waiver of bridging studies to ease availability of multiple RBP lots and in order to facilitate common development programmes.

6. Regulatory agencies should consider standardizing the number of RBP lots for development and establish an agency for the timely supply of RBP with varied drug substance lots.

Supplementary Information The online version of this article (https:// doi.org/10.1007/s40290-021-00395-8) contains supplementary material, which is available to authorized users.

Acknowledgements The authors wish to acknowledge the invaluable contribution of Mr Vivian Fritelli, Generics and Biosimilar Medicines, Southern Africa for connecting us with the industry experts. In addition, we would like to thank Dr Akshaya Odak (India), Dr Jayapala Reddy (Brazil), Karun Gaur (Mexico), Nuray Copur Acar, (Turkey) and Satyakumar Punia (Russia) for their constant support in identifying industry expertise and Dr Shobhna Aanand, Metina PharmConsulting, for her contribution to the reviewing of this manuscript.

\section{Declarations}

Funding No grant or financial contribution was received from a third party. The open access fee was paid by the authors.

Conflict of interest HR, AS, CL, LL and SS declare that this study was conducted in the absence of any commercial or financial relationships that could be considered as a potential conflict of interest.
Availability of data and material A copy of the anonymized data related to the questionnaire responses are available upon reasonable request to the authors.

Code availability Not applicable.

Author contributions HR: designing of research methodology; questionnaire development; the study sample selection; online interviews; data collection; statistical analysis; interpretation and writing of the first draft of the manuscript. CL: reviewing of the questionnaire, support in identifying appropriate participants for the study; and review of the first draft of the manuscript. AS: review of the questionnaire, support in identifying appropriate participants for the study, review of the first draft of the manuscript. LL: reviewing of the questionnaire, support in identifying appropriate participants for the study; and review of the first draft of the manuscript. SS: development of the concept; supervision of the study; review of the questionnaire; research coordination; interpretation of the results; and review of the first and final draft of the manuscript. All authors are responsible for correctness of the statements provided in the manuscript.

Ethical approval The study has been approved by the Health, Science, Engineering and Technology ECDA, University of Hertfordshire. Protocol number-aLMS/PGR/UH/03332(1).

Consent to participate Written informed consent was obtained from participants prior to filling in the questionnaire.

Consent for publication Not applicable.

Open Access This article is licensed under a Creative Commons Attribution-NonCommercial 4.0 International License, which permits any non-commercial use, sharing, adaptation, distribution and reproduction in any medium or format, as long as you give appropriate credit to the original author(s) and the source, provide a link to the Creative Commons licence, and indicate if changes were made. The images or other third party material in this article are included in the article's Creative Commons licence, unless indicated otherwise in a credit line to the material. If material is not included in the article's Creative Commons licence and your intended use is not permitted by statutory regulation or exceeds the permitted use, you will need to obtain permission directly from the copyright holder. To view a copy of this licence, visit http://creativecommons.org/licenses/by-nc/4.0/.

\section{References}

1. Davies DN. The future of biologics. 2017. https://www.theph armaletter.com/article/the-future-of-biologics. Accessed 12 Jan 2021.

2. Aitken M. Biologics market dynamics: setting the stage for biosimilars. FDA/FTC Workshop on a Competitive Marketplace for Biosimilar. 2020. https://www.ftc.gov/system/files/documents/ public_events/1568297/aitken_-_biologics_market_dynamics_ setting_the_stage_for_biosimilars_slides.pdf. Accessed 12 Jan 2021.

3. Centers for Medicare and Medicaid Services. Medicare Part B Drug Average Sales Price. 2019. https://www.cms.gov/Medicare/ Medicare-Fee-for-Service-Part-B-Drugs/McrPartBDrugAvgSales Price. Accessed 12 Jan 2021.

4. US Food and Drug Administration. Biosimilar and Interchangeable Products. 2017. https://www.fda.gov/drugs/biosimilars/biosi milar-and-interchangeable-products. Accessed 12 Jan 2021. 
5. Biosimilar Council. White Paper Part 2: Failure to launch: Barriers to Biosimilar Market Adoption. 2019. https://www.biosimilar scouncil.org/wp-content/uploads/2019/09/AAM-BiosimilarsCouncil-Failure-to-Launch-2-web.pdf. Accessed 12 Jan 2021.

6. Kent D, Rickwood S, Biase SD. Disruption and maturity: The next phase of biologics. 2017. p. 1-24. https://www.iqvia.com/-/ media/iqvia/pdfs/nemea/uk/disruption_and_maturity_the_next_ phase_of_biologics.pdf. Accessed 12 Jan 2021.

7. Wroblewsk MS, Jex EA, Munck SD, Garmon CJ, Michel ST, et al. Emerging Health Care Issues: Follow-on Biologic Drug Competition. Federal Trade Commission Report. 2009. p. 1-120. https://www.ftc.gov/sites/default/files/documents/reports/emerg ing-health-care-issues-follow-biologic-drug-competition-federaltrade-commission-report/p083901biologicsreport.pdf. Accessed 12 Jan 2021.

8. Kabir ER, Moreino SS, Siam MKS. The breakthrough of biosimilars: a twist in the narrative of biological therapy. Biomolecules. 2019;9:1-34. https://doi.org/10.3390/biom9090410.

9. Rahalkar H, Cetintas HC, Salek S. Quality, non-clinical and clinical considerations for biosimilar monoclonal antibody development: EU, WHO, USA, Canada, and BRICS-TM regulatory guidelines. Front Pharmacol. 2018;9:1-17. https://doi.org/10. 3389/fphar.2018.01079.

10. Visiongain. Pharma Leader Series: 25 Top Biosimilar Drug Manufacturers 2016-2026. 2016. https://www.visiongain.com/ report/pharma-leader-series-25-top-biosimilar-drug-manufactur ers-2016-2026/. Accessed 12 Jan 2021.

11. MP Team. Global Biosimilars Outlook-2019 \& beyond. 2019. p. 1-27. https://mpadvisor.com/tocDocument/MP\%20Group\%20-\% 20Global\%20Biosimilars\%20Outlook\%202019.pdf. Accessed 12 Jan 2021.

12. Gautam A. Strategies for biosimilars in emerging markets. Nat Rev Drug Discov. 2017;16:520-1. https://doi.org/10.1038/nrd. 2017.113.

13. USP convention. Guiding Principles for Coining United States Adopted Names for Drugs (2016 USP Dictionary of USAN and International Drug Names). 2016. http://www.uspusan.com/usan/ pub/index 1.html. Accessed 14 June 2021.

14. CDER, CBER. Nonproprietary Naming of Biological Products Guidance for Industry.2017. https://www.fda.gov/files/drugs/publi shed/Nonproprietary-Naming-of-Biological-Products-Guidancefor-Industry.pdf.

15. ANVISA. RESOLUÇÃO DA DIRETORIA COLEGIADA-RDC $\mathrm{N}^{\mathrm{o}}$ 55, DE 16 DE DEZEMBRO DE 2010. 2010. p. 1-27. http:// antigo.anvisa.gov.br/documents/10181/2718376/\%281\%29RDC_ 55_2010_COMP.pdf/41ebae78-5742-4060-9bec-6ccece9ce262.

16. Russian Federation. Registration dossier for finished medical product. 2014.

17. Department of Biotechnology (DBT), Central Drugs Standard Control Organization (CDSCO). Guidelines on similar biologics: Regulatory Requirements for Marketing Authorization in India. 2016. p. 1-53. http://dbtindia.gov.in/sites/default/files/uploadfiles/ Guidelines_on_Similar_Biologics\%2C2016.pdf.

18. CFDA. Technical Guidelines for R\&D and Evaluation of Biosimilar (Trial). 2014

19. Department of Health, Medicines Control Council (MCC). Biosimilar medicines quality, non-clinical and clinical requirements. 2.30_Biosimilars_Aug14_v3.2014.p. 1-20. https://www.sahpra. org.za/wp-content/uploads/2020/01/d259816c2.30_Biosimilars_ Aug14_v3.pdf.

20. TITCK. Guideline on Biosimilar Medicinal Products. 2017. p. 1-30. https://issuu.com/sarfarazniazi/docs/turkishbiosimilargui dances. Accessed 12 Jan 2021.

21. COFEPRIS. Official Mexican Standard NOM-257-SSA1-2014, Biotechnological Medications. Federal Committee for Protection from Sanitary Risks. 2014.
22. Arias A. Biosimilars and Biobetters: Market Trends and Future Opportunities. IQVIA European Thought Leadership, 2020 September. https://event.on24.com/eventRegistration/EventLobby Servlet?target $=$ reg20.jsp\&partnerref $=I N N S i g h t \&$ eventid $=26460$ 86\&sessionid=1\&key=E57E9B57D61A16E9AE19A38931236B $76 \&$ regTag $=\&$ sourcepage $=$ register $\& u t m \_$campaign $=2020$ ArkNewsletterOct_GBU_TC_SD\&utm_medium=email\&utm_ source=Eloqua. Accessed 14 Jan 2021.

23. Singh P, Anasari AF, Bhamare B, Vishwasrao S, Kokil S. Developing Biosimilars in Emerging Markets: Regulatory and Clinical Considerations. National Conference on Current Challenges of Biopharmaceuticals, Vol.1.2016. https://www.researchgate.net/ publication/301214317_Developing_Biosimilars_in_Emerging Markets_Regulatory_and_Clinical_Considerations._Accessed 12 Jan 2021.

24. Welch AR. What to Know About Emerging Market Biosimilar Pathways? Biosimilar Development. 2016. https://www.biosi milardevelopment.com/doc/what-to-know-about-emerging-market-biosimilar-pathways-0001 Accessed 12 Jan 2021.

25. McKinsey \& Company. Five things to know about biosimilars right now. McKinsey \& Company. 2018. https://www.mckinsey. com/industries/pharmaceuticals-and-medical-products/our-insig hts/five-things-to-know-about-biosimilars-right-now.

26. Webster CJ, Woollett GR. A "global reference" comparator for biosimilar development. BioDrugs. 2017;31(4):279-86. https:// doi.org/10.1007/s40259-017-0227-4.

27. International generic and biosimilar medicines association (IGBA). The Importance of Global Regulatory Harmonization for Biosimilar Medicines. In: IGBA Conference. 2019. p. 1-47.

28. Ferreri D. WHO Survey Identifies Global Regulatory Challenges for Biosimilars. AJMC. 2020. https://www.centerforbiosimilars. $\mathrm{com} /$ view/who-survey-identifies-global-regulatory-challenges-forbiosimilars. Accessed 12 Jan 2021.

29. Chen Y, Dikan J, Heller J, da Silva JS. McKinsey \& Company. Five things to know about biosimilars right now. McKinsey \& Company. 2018. https://www.mckinsey.com/industries/pharm aceuticals-and-medical-products/our-insights/five-things-toknow-about-biosimilars-right-now. Accessed 12 Jan 2021.

30. Webster CJ, Wong AC, Woollett GR. An efficient development paradigm for biosimilars. BioDrugs. 2019;33:603-11. https://doi. org/10.1007/s40259-019-00371-4.

31. International generic and biosimilar medicines association (IGBA). Developing a Regulatory Policy Framework Supporting Biosimilar Competition: The Opportunity for Tailored Clinical Biosimilar Development. IGBA Conference, 2020. p. 1-6. https:// www.igbamedicines.org/doc/IGBA\%20Biosimilars\%20Clinical\% 20Trial\%20Tailoring\%20policy\%20paper\%20Sept2020\%20rev ision02.pdf. Accessed 12 Jan 2021.

32. World Health Organisation (WHO). Guidelines on Evaluation of Similar Biotherapeutic Products (SBPs), Annex 2, WHO TRS No. 977, 2009. https://www.who.int/biologicals/areas/biological_thera peutics/BIOTHERAPEUTICS_FOR_WEB_22APRIL2010.pdf.

33. World Health Organization (WHO). Annex 2: Guidelines on evaluation of monoclonal antibodies as similar biotherapeutic products (SBPs). 2017. p. 51-89. https://www.who.int/biolo gicals/biotherapeutics/WHO_TRS_1004_web_Annex_2.pdf? ua=1. WHO Technical Report Series, No. 1004, 2017.

34. World Health Organization (WHO). WHO Questions and Answers: Similar Biotherapeutic Products. 2017. p. 1-30. https:// www.who.int/biologicals/expert_committee/QA_for_SBPs_ ECBS_2018.pdf?ua=1.

35. Cazap E, Jacobs I, Mcbride A, Popovian R, Sikora K. Global acceptance of biosimilars: importance of regulatory consistency, education, and trust. Oncologist. 2018;23(10):1188-98. https:// doi.org/10.1634/theoncologist.2017-0671. 
36. McCamish M, Woollett G. Worldwide experience with biosimilar development. MAbs. 2011;3(2):209-17. https://doi.org/10.4161/ mabs.3.2.15005.

37. Gupta SK, Chaudhari PS, Nath R. Opportunities and Challenges in Biosimilar Development. Bioprocess International. 2017. https://bioprocessintl.com/manufacturing/biosimilars/opportunit ies-challenges-biosimilar-development/. Accessed 14 June 2021.

38. Griffiths E. Quality standards for biopharmaceuticals: the importance of good manufacturing practice. GaBI. 2020;9(3):97-9, 124. https://doi.org/10.5639/gabij.2020.0903.017.
39. Sia CH, Sia MK, Chan LW. Global challenges in the manufacture, regulation and international harmonization of GMP and quality standards for biopharmaceuticals. GaBI. 2020;9(2):52-63. https:// doi.org/10.5639/gabij.2020.0902.010.

40. Vulto AG, Jaquez OA. The process defines the product: what really matters in biosimilar design and production? Rheumatology (Oxford). 2017;56(suppl_4):iv14-29. https://doi.org/10.1093/ rheumatology/kex278. 